\title{
Can Life Prior To Death Predict Quality of Death Among Older Chinese? A Retrospective Cohort Study
}

Jing LI

Fudan University

Liangjun Song ( $\nabla$ songliangjun@fudan.edu.cn )

Fudan University

Xizhe Peng

Fudan University

Zhan Hu

Fudan University

\section{Research Article}

Keywords: older people, life prior to death, quality of death, morbidity, activities of daily living (ADLs)

Posted Date: January 20th, 2022

DOI: https://doi.org/10.21203/rs.3.rs-1226506/v1

License: (c) (i) This work is licensed under a Creative Commons Attribution 4.0 International License. Read Full License 


\section{Abstract \\ Background}

The pursuit of a good death is crucial in aging societies. This retrospective cohort study investigated the relation between life prior to death and quality of death among older Chinese.

\section{Methods}

End-of-life data reported by relatives of participants (aged 54 and over) from the Chinese Longitudinal Healthy Longevity Survey (CLHLS) who deceased between January 2011 and June 2019 were utilised. Life prior to death included health condition (morbid or not) and physical functioning (a latent factor with six indicators). Quality of death was assessed by painlessness and consciousness at death.

Confirmatory Factor Analysis was employed to examine the factor structure of physical functioning and Structural Equation Modeling to explore associations between life prior to death and death quality based on sex and rural/urban residence.

\section{Results}

Freedom from chronic diseases was found to contribute to high-quality deaths (i.e., being painless and conscious) both directly and indirectly by enhancing physical functioning. Men and women diverged towards end-of-life: women were moderately less liable to illnesses and thus less painful at death. Yet, men functioned much better, and more often remained conscious when dying. Location's effect was weaker: although rural residents were more prone to painful deaths than urban dwellers, this urban-rural divide was slightly narrowed by rural settlers' relative health, which also indirectly led to their slight advantage in consciousness at death.

\section{Conclusions}

The results suggested that different dimensions of life prior to death predicted quality of death. Additionally, morbidity's effect on functioning and death quality stresses health management's role in improving end-of-life experiences.

\section{Background}

Improving end-of-life experiences is essential to successful aging [1]. According to practitioners and health researchers, a high quality of life prior to death emphasizes healthier survival (freedom from illness, or management of pain and other unpleasant symptoms from chronic diseases) and satisfactory functional status (autonomy in activities of daily living, or ADLs) given expected proximity to death [2, 3 , 4]. Additionally, to 'die well' is increasingly acknowledged as crucial in aging societies $[5,6,7]$. Quality 
markers of a good death identified in empirical studies include freedom from pain or discomfort, being conscious and alert, feeling dignified and prepared, etc. [1, 3, 5, 8]. So far, much end-of-life research has centred on patients diagnosed with terminal illnesses $[2,9]$. Despite providing substantial clinical and policy implications, this line of inquiry neglects older people who are relatively healthy and functionally capable.

Research on late life in China, instead, has examined experiences of older adults in general to promote healthy longevity for all. After all, this is a particular challenge for the country given that the proportion of its older population (aged 60 or more) is projected to increase by $28 \%$ ( 402 million) by 2040 , a faster rate than most countries across the world [10]. Consistent with previous research conducted elsewhere, indicators for healthy aging in China also comprise being disease-free and able to perform ADLs independently, among others $[11,12]$. Moreover, older Chinese females appear relatively disadvantaged in health and functioning, a phenomenon prevailing in contemporary human societies [13], although some studies conducted in China did detect male disadvantages in certain diseases [14] or equal functional statuses for men and women [15]. Furthermore, adopting urban-rural residence as a proxy for socioeconomic status (SES) in China, scholars have found that urban settlers are more likely to be morbid or multimorbid, while rural residents face greater risks of functional disability $[11,12,16,17]$. Such urban-rural disparities seem paradoxical, as morbidity/multimorbidity is repeatedly found to be correlated with functional limitation $[15,18]$. Reasons for these sex- and residence-related nuances might be nosologic, methodological, or substantive: definitions and classifications of diseases might affect the measure of morbidity [13], the approximation of functional limitation by observed scores (e.g., difficulties in one or more $A D L$ items, or sums of scores from ADL items) can be inaccurate because of measurement error in ADL items $[12,18]$, and the unequal distribution of medical resources across regions may lead to the under-report and underdiagnosis of diseases in rural villages [14].

Instrumental in promoting understandings of late life, the abovementioned works in China hardly ever associated life prior to death to quality of death. The few exceptions either focused on adverse health conditions at end-of-life or limited their examination of death statuses to death location or level of pain experienced $[7,19,20]$. The effects of health conditions (single disease or multimorbidity) on physical functioning given proximity to death, and how they both could have influenced the quality of death, remain inconclusive. Whether sex or urban-rural residence plays a role towards end-of-life is also largely unclear. Thus, to address these gaps, the current study analysed end-of-life data collected from relatives of older adults (aged 54 and over) participating in the Chinese Longitudinal Healthy Longevity Survey (CLHLS) who deceased between 2011 and 2019. This is the first known retrospective design to capture the mechanisms between life prior to death and death quality, and to explore sex and urban-rural disparities drawing on nationwide data from older Chinese.

\section{Methods}

\section{Data source}


The CLHLS aimed to understand factors associated with mortality and healthy longevity [17]. The baseline survey was conducted in 1998 in one half of the cities and counties randomly selected from 22 out of 31 provinces throughout mainland China [21]. The approximately 985 million people in these participating provinces accounted for $85 \%$ of the Chinese population, revealing the wide coverage of the CLHLS. Respondents were older people aged 65 and over, although the oldest old (80-year-olds and older) and males were oversampled to achieve adequate representation [22]. Seven follow-up surveys were conducted in 2000, 2002, 2005, 2008-09, 2011-12, 2014 and 2017-18, with replacements for those who deceased between waves. Detailed information on sampling procedures and survey protocols are available from Peking University Open Access Research Database (http://opendata.pku.edu.cn/), and ethical approval was gained from the Ethics Committees of Peking University and Duke University [17, 23]. Assessments of data (accuracy of age, consistency, and validity of various measures, etc.) yielded proof of high data quality [22].

After participants had died between waves, a close contact (often a family member, but occasionally, a friend, social worker, or others) was interviewed in the next immediate wave about the decedents' predeath and dying experiences. The information gathered included medical history and functional capacities prior to death, cause of death, death statuses, among others. Data on the 9,778 deaths during the 2011-12 $(n=5,642), 2014(n=2,589)$, and 2017-18 $(n=1,547)$ waves were utilized in this research, because the questions on medical history were changed since the 2011-12 wave, rendering it unfeasible to incorporate the data from earlier waves.

\section{Measurement}

\section{Life prior to death}

Life prior to death consisted of health condition and physical functioning. Health condition was measured by the number of known diseases the departed had approximately three months or less prior to death, where a code of 0 stood for no disease at all, 1 for one disease, and 2 for two diseases and more (i.e., multimorbidity). The diseases reported were common chronic illnesses among older Chinese, which included hypertension, diabetes, heart diseases, cerebrovascular disease (CVD), bronchitis, emphysema, pneumonia, asthma, tuberculosis, cancer, glaucoma, cataracts, gastric or duodenal ulcer, Parkinson's Disease, bedsores, dementia, and arthritis.

To account for measurement errors in observed variables, physical functioning was conceptualized as a latent factor with six indicators, all of which corresponded to the decedents' ability to perform six ADLs (bathing/showering, dressing, getting to the toilet, indoor mobility, continence, and eating) within three months before their deaths. The answers from the surrogate respondents (relatives or other close contacts) were dichotomized into 'dependent $(=0)$ ' and 'independent $(=1)$ '.

\section{Quality of death}


Quality of death was assessed by two items from the CLHLS: whether the older adults seemed painful at death, and whether they lost consciousness when dying. The former was evaluated by a five-point Likert scale ranging from 1 ('very painful') to 5 ('peaceful'). The latter had two categories: 1 for being conscious and 2 if otherwise. During data analyses, responses to both items were re-coded as 0 when they were negative ('very painful' and 'relatively painful'; 'unconscious') and 1 when positive ('all right', 'relatively peaceful' and 'peaceful'; 'conscious').

\section{Covariates}

Sex and residence location were the covariates in this study. Both variables were dichotomous: (a) sex (0 $=$ 'male', 1 = 'female'), and $(\mathrm{b})$ residence location ( 0 = 'rural areas', 1 = 'urban areas').

\section{Data Analyses}

Confirmatory Factor Analysis (CFA) was adopted to examine the factor structure of the latent constructphysical functioning. The following indices were utilized to determine the model fit: statistical nonsignificance of model chi-square ( $a$ at .05 ), the comparative fit index (CFI) $\geq .95$, the Tucker-Lewis Index $(T L I) \geq .95$, the standardized root mean residual $(S R M R) \leq .08$, the root mean square error of approximation (RMSEA) <.07 [24].

Subsequently, to isolate the effects of sex and urban-rural residence at the observed level (i.e., in each of the six indicators of physical functioning), the 'multiple indicators, multiple causes' (MIMIC) method was applied $[25 ; 26]$. To the original CFA model, a hypothesized path linking a covariate (sex or residence) and one indicator of physical functioning (bathing, dressing, etc.) was added, while holding constant the level of physical functioning as a latent factor. This procedure was repeated for all six indicators one-by-one, and for both covariates (sex and residence). A good overall model fit, in addition to a statistically significant regression coefficient for the added path, would provide evidence of the covariate's effect irrespective of the latent construct. Any path satisfying both criteria would be retained in the model for subsequent analysis.

Finally, Structural Equation Modelling (SEM) was employed to identify associations between life prior to death and quality of death based on sex and residence. Both CFA and SEM were conducted with Mplus 8.3, the software program designed for latent variable modelling [27].

\section{Results}

\section{Descriptive statistics}

Table 1 below exhibits socio-demographic characteristics of the decedents. Age at death ranged between 54 and $121(M=94.33, S D=7.95)$. Women comprised $58.75 \%$ of the sample. On average, the participants received 1.48 years of schooling $(S D=2.91)$. Towards end-of-life, over half $(58.68 \%)$ lived in rural regions while the rest in cities or towns. Most subjects (87.03\%) were community-dwelling, and about $90 \%$ were 
primarily cared for by their family. Additionally, as far as the available data have implied, nine out of ten responses were from a next of kin. Given that information on end-of-life status tends to be accurate when provided by family members $[7,28]$, the proxy responses from the CLHLS were considered reliable.

Table 1

Socio-demographic characteristics of subjects

\begin{tabular}{|c|c|c|c|c|c|}
\hline Characteristics & $N$ & $\%$ & Characteristics & $N$ & $\%$ \\
\hline \multicolumn{3}{|l|}{ Age at death (years) } & \multicolumn{3}{|c|}{$\begin{array}{l}\text { Relationship between the respondent and the } \\
\text { deceased* }\end{array}$} \\
\hline Mean $(S D)$ & \multicolumn{2}{|c|}{$94.35(9.03)$} & Relative & 1,106 & 90.07 \\
\hline Minimum-maximum & \multicolumn{2}{|c|}{$54-121$} & Non-relative & 122 & 9.93 \\
\hline \multicolumn{3}{|l|}{ Sex } & \multicolumn{3}{|c|}{ Months between the last interview and death } \\
\hline Male & 4,033 & 41.25 & Mean $(S D)$ & \multicolumn{2}{|c|}{$18.33(11.29)$} \\
\hline Female & 5,745 & 58.75 & Minimum-maximum & \multicolumn{2}{|c|}{$0-61.70$} \\
\hline \multicolumn{3}{|l|}{ Education (years) } & \multicolumn{3}{|l|}{ Primary caregiver } \\
\hline Mean $(S D)$ & \multicolumn{2}{|c|}{$1.48(2.91)$} & No caregiver needed & 233 & 2.42 \\
\hline Minimum-maximum & \multicolumn{2}{|l|}{$0-25$} & Lack of a caregiver & 66 & .69 \\
\hline \multicolumn{3}{|c|}{ Residence location prior to death } & Spouse & 751 & 7.80 \\
\hline Rural areas & 5,738 & 58.68 & Children or grandchildren & 7,899 & 82.08 \\
\hline $\begin{array}{l}\text { Urban areas } \\
\text { (cities/towns) }\end{array}$ & 4,040 & 41.32 & Others from family, or friends & 239 & 2.48 \\
\hline \multicolumn{3}{|c|}{ Marital status prior to death } & Others & 435 & 4.52 \\
\hline Unmarried & 7,719 & 80.11 & Place of death & & \\
\hline Married & 1,916 & 19.89 & Home & 8,544 & 88.74 \\
\hline \multicolumn{3}{|c|}{ Living arrangement prior to death } & Hospital & 812 & 8.43 \\
\hline Alone & 1,245 & 12.97 & Nursing homes & 215 & 2.23 \\
\hline With others & 8,352 & 87.03 & Others & 57 & .59 \\
\hline
\end{tabular}

Note. $N=9,778 .{ }^{*}$ Information on respondents' relationship to the deceased was only available for the 2018 wave.

Table 2 here describes health condition, six indicators of physical functioning, and two items representing quality of death among participants. Over half of the total sample were morbid (57.43\%). Difficulties with performing ADLs were prevalent (between $65.62 \%$ and $79.64 \%$ ) in five out of six activities except for 
continence, with which slightly less than half $(48.10 \%)$ had trouble. Almost two fifths of the deaths were of less-than-satisfactory qualities, featured by feelings of pain (38.59\%) and unconsciousness (36.09\%). 
Table 2

Descriptive statistics on health condition, physical functioning, and quality of death by sex and location

Sex

Male Female

$N(\%) \quad N(\%)$
Residence location

Rural areas

Urban areas

$N(\%) \quad N(\%)$

Health condition $(N$ in total

sample; \%)

No disease $(4,026 ; 42.58)$

1,455

$2,571(46.21) \quad 2,523(45.22)$

1,503

(37.38)

$(38.77)$

Single disease $(2,932 ; 31.01)$

1,282

(32.94)

$1,650(29.65) \quad 1,713(30.70)$

1,219

(31.44)

Two or more $(2,498 ; 26.42)$

1,155

(29.68)

$1,343(24.14) \quad 1,343(24.07)$

1,155

(29.79)

Physical functioning

Bathing/Showering ( $\mathrm{Nin}$ total sample; \%)

Independent $(1,944 ; 20.36)$

$977(24.89)$

$967(17.21)$

$1,204(21.36)$

740

(18.93)

Dependent $(7,602 ; 79.64)$

2,949

(75.11)

$4,653(82.79) \quad 4,433(78.64)$

3,169

$(81.07)$

Getting dressed and undressed ( $N$ in total sample; \%)

Independent $(2,615 ; 27.39)$

1,227

$(31.20)$

$1,388(24.72) \quad 1,546(27.45)$

1,069

$(27.31)$

Dependent $(6,932 ; 72.61)$

2,706

$(68.80)$

$4,226(75.28)$

$4,087(72.55)$

2,845

$(72.69)$

Getting to the toilet and cleaning ( $N$ in total sample; \%)

Independent $(2,469 ; 25.77)$

1,176

(29.82)

1,293 (22.94)

$1,457(25.76)$

1,012

(25.80)

Dependent $(7,111 ; 74.23)$

2,768

$4,343(77.06)$

$4,200(74.24)$

2,911

(70.18)

$(74.20)$

Indoor mobility ( $N$ in total sample; \%)

Independent $(2,395 ; 25.06)$

1145

(29.13)

$1,250(22.21) \quad 1,434(25.39)$

961

(24.58)

Dependent $(7,162 ; 74.94)$

2785

(70.87)

$4,377(77.79)$

$4,213(74.61)$

2,949

$(75.42)$

Controlling bladder/bowel functions ( $N$ in total sample; \%)

Note. Percentages (\%) are proportions of non-missing values. 


\begin{tabular}{|c|c|c|c|c|}
\hline \multirow[b]{2}{*}{ Independent $(4,966 ; 51.90)$} & \multirow{2}{*}{$\begin{array}{l}\text { Sex } \\
\qquad \begin{array}{l}2,102 \\
(53.45)\end{array}\end{array}$} & \multicolumn{2}{|c|}{ Residence location } & \multirow[b]{2}{*}{$\begin{array}{l}1,947 \\
(49.68)\end{array}$} \\
\hline & & $2,864(50.83)$ & $3,019(53.44)$ & \\
\hline Dependent $(4,602 ; 48.10)$ & $\begin{array}{l}1,831 \\
(46.55)\end{array}$ & $2,771(49.17)$ & $2,630(46.56)$ & $\begin{array}{l}1,972 \\
(50.32)\end{array}$ \\
\hline \multicolumn{5}{|c|}{ Feeding oneself ( $N$ in total sample; \%) } \\
\hline Independent $(3,284 ; 34.38)$ & $\begin{array}{l}1,483 \\
(37.74)\end{array}$ & $1,801(32.03)$ & $1,949(34.54)$ & $\begin{array}{l}1,335 \\
(34.15)\end{array}$ \\
\hline Dependent $(6,268 ; 65.62)$ & $\begin{array}{l}2,446 \\
(62.26)\end{array}$ & $3,822(67.97)$ & $3,694(65.46)$ & $\begin{array}{l}2,574 \\
(65.85)\end{array}$ \\
\hline \multicolumn{5}{|l|}{ Quality of death } \\
\hline \multicolumn{5}{|c|}{ Freedom from pain ( $N$ in total sample; \%) } \\
\hline Yes $(5,475 ; 61.41)$ & $\begin{array}{l}2,087 \\
(56.94)\end{array}$ & $3,388(64.52)$ & $3,121(59.39)$ & $\begin{array}{l}2,354 \\
(64.30)\end{array}$ \\
\hline No $(3,441 ; 38.59)$ & $\begin{array}{l}1,578 \\
(43.06)\end{array}$ & $1,863(35.48)$ & $2,134(40.61)$ & $\begin{array}{l}1,307 \\
(35.70)\end{array}$ \\
\hline \multicolumn{5}{|l|}{$\begin{array}{l}\text { Being conscious ( } N \text { in total } \\
\text { sample; \%) }\end{array}$} \\
\hline Yes $(6,085 ; 63.91)$ & $\begin{array}{l}2,624 \\
(66.96)\end{array}$ & $3,461(61.78)$ & $3,646(64.88)$ & $\begin{array}{l}2,439 \\
(62.52)\end{array}$ \\
\hline No $(3,436 ; 36.09)$ & $\begin{array}{l}1,295 \\
(33.04)\end{array}$ & $2,141(38.22)$ & $1,974(35.12)$ & $\begin{array}{l}1,462 \\
(37.48)\end{array}$ \\
\hline
\end{tabular}

Sex differences emerged towards end-of-life. Women were relatively heathier, as the proportion of women whose close contacts recalled having no disease $(46.21 \%)$ was larger than that of men (37.38\%), while a higher percentage of men were believed to be morbid. On the other hand, men functioned much better, because a higher fraction of men than women could perform all ADLs without assistance: the sex gap regarding proportions varied between $2.62 \%$ and $7.68 \%$. Discrepancies persisted till death. Painlessness was more often witnessed among female decedents ( $64.52 \%$ of women but $56.94 \%$ of men), while consciousness among male ones $(66.96 \%$ vs. $61.78 \%)$.

Compared to sex disparities, residence-based divergences were not as pronounced. Urban dwellers were more often morbid: within three months prior to death, $31.44 \%$ of city-settlers lived with one disease and $29.79 \%$ with two or more, while the respective figures were $30.70 \%$ and $24.07 \%$ for rural residents. Circumstances with physical functioning (indicated by six ADL measures) were less straightforward. Higher percentages of older people in urban areas could perform three ADLs independently than their peers in rural locations (bathing/showering, indoor mobility, and continence), and the percentage 
differences ranged from .81-3.76\%. By comparison, urban-rural divides in the remaining three ADLs (dressing, getting to the toilet, and eating) were minimal-percentage differences at .39\% and lower. When dying, a larger share of urban decedents was painless ( $64.30 \%$ vs. $59.39 \%)$, but proportions of consciousness at death were substantially similar in urban and rural regions $(62.52 \%$ in cities or towns and $64.88 \%$ in villages).

\section{Cfa And Mimic-model Method}

CFA was carried out to determine whether a common factor, physical functioning, underlay the six items of ADLs (see the graphic representation in Figure 1, Panel A). All fit indices were optimal (CFI= 1.000; TLI= 1.000; RMSEA $=.038,90 \% \mathrm{Cl}:[.033, .044]$; SRMR $=.010)$, apart from the statistically significant chi-square statistics $\left(\chi^{2}\right.$ [df] = 135.319 [9], $\left.p<.001\right)$. Because model chi-square tests the exact-fit hypothesis, which is sensitive to sample sizes larger than 300 , the specified model was still recognized as a reasonably good fit for the data [29].

The MIMIC-method was then applied to identify the effect of covariates (sex and residence) at the observed level (i.e., in one or more indicators of physical functioning; see Figure 1, Panel B for an example) while holding constant the latent factor. The results were summed in Table 3 below (values of other path coefficients are substantially similar with the original CFA model and are thus omitted to save space but are available on request). The following trends emerged: proxy respondents of older women were more inclined to recall problems with bathing/showering and indoor mobility, but surrogates of older men were more liable to report issues with continence and feeding. In terms of location, surrogate respondents for rural settlers noticed more difficulties with dressing and getting to the toilet, while those for urban residents suggested more trouble with bathing/showering and continence. These four paths were retained in the subsequent SEM analysis. 
Table 3

Fit indices and slope coefficients for MIMIC models testing sex-related or residence-based response differences on each indicator of physical functioning

\begin{tabular}{|c|c|c|c|c|c|c|c|c|}
\hline Indicator & $\chi^{2} S B$ & df & $\begin{array}{l}\text { RMSEA } \\
\text { [90\% Cl] }\end{array}$ & CFI & TLI & $b$ & $\beta$ & $p$ \\
\hline \multicolumn{9}{|l|}{ Sex } \\
\hline Bathing/Showering & 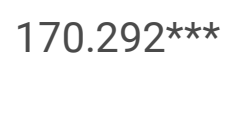 & 13 & $\begin{array}{l}.036[.031, \\
.040]\end{array}$ & 1.000 & .999 & $-096 * \star \star$ & $-.047 \star \star \star$ & $\begin{array}{l}< \\
.001\end{array}$ \\
\hline Dressing & $189.440 * \star \star$ & 13 & $\begin{array}{l}.038[.033, \\
.042]\end{array}$ & 1.000 & .999 & -.002 & -.001 & .889 \\
\hline Getting to the toilet & $187.955^{\star \star \star}$ & 13 & $\begin{array}{l}.037[.033, \\
.042]\end{array}$ & 1.000 & .999 & -.023 & -.011 & .065 \\
\hline Mobility & $187.677^{\star \star \star}$ & 13 & $\begin{array}{l}.037[.033, \\
.042]\end{array}$ & 1.000 & .999 & $-.029 *$ & $-.014^{\star}$ & .031 \\
\hline Continence & 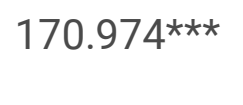 & 13 & $\begin{array}{l}.036[.031, \\
.040]\end{array}$ & 1.000 & .999 & $.106^{\star \star \star}$ & $.052^{\star \star \star}$ & $\begin{array}{l}< \\
.001\end{array}$ \\
\hline Feeding & $188.831^{\star \star \star}$ & 13 & $\begin{array}{l}.038[.033, \\
.042]\end{array}$ & 1.000 & .999 & $.034^{\star}$ & $.017 \star$ & .046 \\
\hline \multicolumn{9}{|l|}{ Residence location } \\
\hline Bathing/Showering & 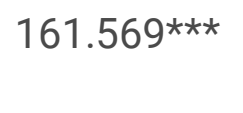 & 13 & $\begin{array}{l}.035[.030, \\
.039]\end{array}$ & 1.000 & .999 & $-.060 \star \star$ & $-.029 * \star$ & .002 \\
\hline Dressing & $162.569 * \star \star$ & 13 & $\begin{array}{l}.035[.030, \\
.039]\end{array}$ & 1.000 & .999 & $.040^{\star \star}$ & $.019 \star \star$ & .004 \\
\hline Getting to the toilet & $161.153^{\star \star *}$ & 13 & $\begin{array}{l}.034[.030, \\
.039]\end{array}$ & 1.000 & .999 & $.046^{\star \star \star}$ & $.023^{\star \star \star}$ & $\begin{array}{l}< \\
.001\end{array}$ \\
\hline Mobility & $166.752^{\star \star \star}$ & 13 & $\begin{array}{l}.035[.030, \\
.040]\end{array}$ & 1.000 & .999 & .012 & .006 & .384 \\
\hline Continence & $165.912^{\star \star \star}$ & 13 & $\begin{array}{l}.035[.030, \\
.040]\end{array}$ & 1.000 & .999 & $-.075^{\star \star}$ & $-.037 \star \star$ & .001 \\
\hline Feeding & $166.747^{\star \star \star}$ & 13 & $\begin{array}{l}.035[.030, \\
.040]\end{array}$ & 1.000 & .999 & .029 & .014 & .090 \\
\hline
\end{tabular}

\section{SEM}

SEM was performed to analyse the associations between life prior to death and quality of death. As depicted in Figure 2, the model fitted the data well, $\chi^{2}(\mathrm{df})=293.502(26), p<.001 ; \mathrm{CFI}=.999$; TLI= .999; RMSEA $=.033,90 \% \mathrm{Cl}:[.029, .036] ;$ SRMR $=.015$. Both unstandardized estimates $(b)$ and standardized 
estimates $(\beta)$ for the hypothesized paths are displayed in Table 4, and Table 5 (p.18) summarises the direct, indirect, and total effects between variables/factors in interest. 
Table 4

Parameter estimates for the associations between life prior to death and quality of death

\begin{tabular}{|c|c|c|c|c|c|}
\hline Parameter & $b$ & $S . E_{\cdot b}$ & $\boldsymbol{\beta}$ & $S . E_{\cdot \beta}$ & $p$ \\
\hline \multicolumn{6}{|l|}{ Path coefficient (regression) } \\
\hline 1. $\mathrm{PF} \rightarrow \mathrm{B}$ & 1.000 & .000 & $.961^{\star \star \star}$ & .003 & $<.001$ \\
\hline 2. $P F \rightarrow D$ & $1.745^{\star \star \star}$ & .147 & $.988^{\star \star \star}$ & .002 & $<.001$ \\
\hline 3. $P F \rightarrow G$ & $1.999 * \star \star$ & .241 & $.991 * \star \star$ & .002 & $<.001$ \\
\hline 4. $\mathrm{PF} \rightarrow \mathrm{M}$ & $1.507 * \star \star$ & .107 & $.984 * \star \star$ & .002 & $<.001$ \\
\hline 5. $\mathrm{PF} \rightarrow \mathrm{C}$ & $.385^{\star \star \star}$ & .018 & $.820 * \star \star$ & .007 & $<.001$ \\
\hline 6. $\mathrm{PF} \rightarrow \mathrm{F}$ & $.771^{\star \star \star}$ & .039 & $.945^{\star \star \star}$ & .003 & $<.001$ \\
\hline 7. $\mathrm{HC} \rightarrow \mathrm{PF}$ & $-.736 * \star \star$ & .061 & $-.200 * \star \star$ & .014 & $<.001$ \\
\hline 8. $\mathrm{HC} \rightarrow \mathrm{FP}$ & $-.181^{\star \star \star}$ & .016 & $-.177^{\star \star \star}$ & .016 & $<.001$ \\
\hline 9. $\mathrm{HC} \rightarrow \mathrm{BC}$ & $-.097 * \star \star$ & .017 & $-.089 * * *$ & .015 & $<.001$ \\
\hline 10. $\mathrm{PF} \rightarrow \mathrm{FP}$ & $.023^{\star \star \star}$ & .005 & $.082^{\star \star \star}$ & .017 & $<.001$ \\
\hline 11. $\mathrm{PF} \rightarrow \mathrm{BC}$ & $.115^{\star \star \star}$ & .007 & $.387^{\star \star \star}$ & .015 & $<.001$ \\
\hline 12. Sex $\rightarrow B$ & $-.278^{\star \star \star}$ & .068 & $-.035^{\star \star \star}$ & .009 & $<.001$ \\
\hline 13. Sex $\rightarrow M$ & -.077 & .073 & -.007 & .006 & .292 \\
\hline 14. Sex $\rightarrow C$ & $.172^{\star \star \star}$ & .042 & $.049 \star \star \star$ & .012 & $<.001$ \\
\hline 15. Sex $\rightarrow F$ & $.116^{\star}$ & .055 & $.019 *$ & .009 & .036 \\
\hline 16. Sex $\rightarrow \mathrm{HC}$ & $-.196 * \star \star *$ & .024 & $-.096 * \star \star *$ & .012 & $<.001$ \\
\hline 17. Sex $\rightarrow$ PF & $-.902 * \star \star$ & .108 & $-.120 * \star \star$ & .013 & $<.001$ \\
\hline 18. Sex $\rightarrow$ FP & $.187 * \star \star$ & .028 & $.089 * \star \star$ & .013 & $<.001$ \\
\hline 19. Sex $\rightarrow B C$ & $-.087 * \star$ & .030 & $-.039 * \star$ & .013 & .003 \\
\hline 20. Residence location $\rightarrow B$ & $-.261 * \star$ & .079 & $-.033^{\star *}$ & .010 & .001 \\
\hline 21. Residence location $\rightarrow D$ & .093 & .102 & .007 & .008 & .362 \\
\hline 22. Residence location $\rightarrow G$ & .168 & .107 & .011 & .007 & .112 \\
\hline 23. Residence location $\rightarrow C$ & $-.131^{\star \star}$ & .040 & $-.037 \star \star$ & .011 & .001 \\
\hline
\end{tabular}

Note. S.E., standard error; $b$, unstandardized estimates; $\beta$, standardized estimates; $\mathrm{B}$, bathing/showering; $D$, dressing; $G$, getting to the toilet; $M$, indoor mobility; $C$, continence; $F$, feeding; $H C$, health condition; PF, physical functioning; FP, freedom from pain; BC, being conscious. ${ }^{\star} p<.05$, ${ }^{\star \star} p<$ $.001,{ }^{\star * \star *} p<.001$. 


\begin{tabular}{|c|c|c|c|c|c|}
\hline Parameter & $b$ & $S . E_{\cdot b}$ & $\beta$ & $S . E_{\cdot \beta}$ & $p$ \\
\hline 24. Residence location $\rightarrow \mathrm{HC}$ & $.164^{\star \star \star}$ & .024 & 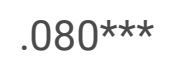 & .012 & $<.001$ \\
\hline 25. Residence location $\rightarrow$ PF & .028 & .098 & .004 & .013 & .778 \\
\hline 26. Residence location $\rightarrow$ FP & 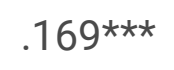 & .028 & $.081^{\star \star \star}$ & .013 & $<.001$ \\
\hline 27. Residence location $\rightarrow \mathrm{BC}$ & -.047 & .029 & -.021 & .013 & .103 \\
\hline \multicolumn{6}{|l|}{ Path coefficient (covariance) } \\
\hline 28. $\mathrm{FP} \leftrightarrow \mathrm{BC}$ & $.113^{\star \star \star}$ & .019 & $.113^{\star \star \star}$ & .019 & $<.001$ \\
\hline \multicolumn{6}{|c|}{$\begin{array}{l}\text { Note. S.E., standard error; } b \text {, unstandardized estimates; } \beta \text {, standardized estimates; } \mathrm{B} \text {, } \\
\text { bathing/showering; } \mathrm{D} \text {, dressing; } \mathrm{G} \text {, getting to the toilet; } \mathrm{M} \text {, indoor mobility; } \mathrm{C} \text {, continence; } \mathrm{F} \text {, feeding; } \mathrm{HC} \\
\text { health condition; } \mathrm{PF} \text {, physical functioning; FP, freedom from pain; } \mathrm{BC} \text {, being conscious. }{ }^{\star} p<.05,{ }^{\star \star} p< \\
.001,{ }^{\star \star \star} p<.001 \text {. }\end{array}$} \\
\hline
\end{tabular}


Table 5

Direct, indirect, and total effects of life prior to death on quality of death

\begin{tabular}{|c|c|c|c|c|c|}
\hline Effect & $b$ & $S . E_{\cdot b}$ & $\boldsymbol{\beta}$ & S.E. ${ }_{\beta}$ & $p$ \\
\hline \multicolumn{6}{|l|}{$\mathrm{HC} \rightarrow \mathrm{FP}$} \\
\hline 1. Total & $-.198 * \star \star$ & .016 & $-.194 * \star \star$ & .015 & $<.001$ \\
\hline 2. Direct & $-.181^{\star \star \star}$ & .016 & $-.177 \star \star \star$ & .016 & $<.001$ \\
\hline 3. Indirect $(\mathrm{HC} \rightarrow \mathrm{PF} \rightarrow \mathrm{FP})$ & $-.017 \star \star \star$ & .004 & $-.016 * \star \star$ & .004 & $<.001$ \\
\hline \multicolumn{6}{|l|}{$\mathrm{HC} \rightarrow \mathrm{BC}$} \\
\hline 4. Total & $-.181^{\star \star \star}$ & .017 & 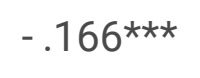 & .015 & $<.001$ \\
\hline 5. Direct & $-.097^{\star \star \star}$ & .017 & $-.089 \star \star \star$ & .015 & $<.001$ \\
\hline 6. Indirect $(\mathrm{HC} \rightarrow \mathrm{PF} \rightarrow \mathrm{BC})$ & $-.085^{\star \star \star}$ & .007 & $-.077 \star \star \star$ & .006 & $<.001$ \\
\hline \multicolumn{6}{|l|}{ Sex $\rightarrow$ PF } \\
\hline 7. Total & $-.757^{\star \star \star}$ & .106 & $-.101^{\star \star \star}$ & .013 & $<.001$ \\
\hline 8. Direct & $-.902^{\star \star \star}$ & .108 & $-.120 \star \star \star$ & .013 & $<.001$ \\
\hline 9. Indirect (sex $\rightarrow \mathrm{HC} \rightarrow \mathrm{PF})$ & $.145^{\star \star \star}$ & .021 & $.019 \star \star \star *$ & .003 & $<.001$ \\
\hline \multicolumn{6}{|l|}{$\mathrm{Sex} \rightarrow \mathrm{FP}$} \\
\hline 10. Total & $.205^{\star \star \star}$ & .028 & $.098^{\star \star \star}$ & .013 & $<.001$ \\
\hline 11. Direct & $.187^{\star \star *}$ & .028 & $.089 \star \star \star$ & .013 & $<.001$ \\
\hline 12. Indirect (total) & $.018^{*}$ & .007 & $.009 *$ & .004 & .013 \\
\hline 13. Indirect $($ sex $\rightarrow H C \rightarrow F P)$ & $.036^{\star \star \star}$ & .005 & $.017 * \star \star$ & .003 & $<.001$ \\
\hline 14. Indirect (sex $\rightarrow$ PF $\rightarrow F P)$ & $-.021^{\star \star \star}$ & .005 & $-.010 * \star \star$ & .002 & $<.001$ \\
\hline 15. Indirect (sex $\rightarrow \mathrm{HC} \rightarrow \mathrm{PF} \rightarrow \mathrm{FP})$ & $.003^{\star \star \star}$ & .001 & $.002^{\star \star \star}$ & .000 & $<.001$ \\
\hline \multicolumn{6}{|l|}{ Sex $\rightarrow B C$} \\
\hline 16. Total & $-.155^{\star \star \star}$ & .030 & $-.069 \star \star \star$ & .013 & $<.001$ \\
\hline 17. Direct & $-.087 * \star$ & .030 & $-.039 * *$ & .013 & .003 \\
\hline 18. Indirect (total) & $-.068 * \star \star$ & .013 & $-.030 \star \star \star$ & .006 & $<.001$ \\
\hline 19. Indirect $(\mathrm{sex} \rightarrow \mathrm{HC} \rightarrow \mathrm{BC})$ & $.019 \star \star \star$ & .004 & $.008^{\star \star \star}$ & .002 & $<.001$ \\
\hline
\end{tabular}

Note. S.E., standard error; $b$, unstandardized estimates; $\beta$, standardized estimates; $\mathrm{B}$, bathing/showering; $D$, dressing; $G$, getting to the toilet; $M$, indoor mobility; $C$, continence; $F$, feeding; $H C$, health condition; PF, physical functioning; FP, freedom from pain; BC, being conscious. ${ }^{\star} p<.05$, ${ }^{\star \star} p<$ $.001,{ }^{\star * \star *} p<.001$. 


\begin{tabular}{|c|c|c|c|c|c|}
\hline Effect & $b$ & $S . E_{\cdot b}$ & $\boldsymbol{\beta}$ & $S . E_{\cdot \beta}$ & $p$ \\
\hline 20. Indirect (sex $\rightarrow$ PF $\rightarrow B C)$ & $-.104 * \star \star$ & .012 & 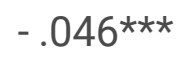 & .005 & $<.001$ \\
\hline 21. Indirect (sex $\rightarrow \mathrm{HC} \rightarrow \mathrm{PF} \rightarrow \mathrm{BC})$ & $.017 * \star \star$ & .002 & $.007 * \star \star$ & .001 & $<.001$ \\
\hline \multicolumn{6}{|l|}{ Residence location $\rightarrow$ PF } \\
\hline 22. Total & -.093 & .098 & -.012 & .013 & .343 \\
\hline 23. Direct & .028 & .098 & -.004 & .013 & .778 \\
\hline 24. Indirect (residence location $\rightarrow \mathrm{HC} \rightarrow \mathrm{PF}$ ) & $-.121^{\star \star \star}$ & .020 & $-.016 * \star \star$ & .003 & $<.001$ \\
\hline \multicolumn{6}{|l|}{ Residence location $\rightarrow$ FP } \\
\hline 25. Total & 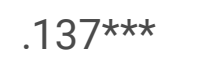 & .028 & $.066^{\star \star \star}$ & .013 & $<.001$ \\
\hline 26. Direct & $.169 * \star \star$ & .028 & $.081^{\star \star \star}$ & .013 & $<.001$ \\
\hline 27. Indirect (total) & 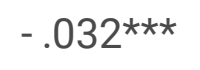 & .006 & $-.015^{\star \star \star}$ & .003 & $<.001$ \\
\hline 28. Indirect (residence location $\rightarrow \mathrm{HC} \rightarrow \mathrm{FP}$ ) & $-.030 \star \star \star$ & .005 & $-.014 * \star \star$ & .002 & $<.001$ \\
\hline 29. Indirect (residence location $\rightarrow$ PF $\rightarrow$ FP) & -.001 & .002 & .000 & .001 & .778 \\
\hline 30. Indirect (residence location $\rightarrow \mathrm{HC} \rightarrow \mathrm{PF} \rightarrow \mathrm{FP}$ ) & $-.003^{\star \star \star}$ & .001 & $-.001 * \star \star$ & .000 & $<.001$ \\
\hline \multicolumn{6}{|l|}{ Residence location $\rightarrow \mathrm{BC}$} \\
\hline 31. Total & $-.074^{\star}$ & .029 & $-.033^{*}$ & .013 & .012 \\
\hline 32. Direct & -.047 & .029 & -.021 & .013 & .103 \\
\hline 33. Indirect (total) & $-.027^{\star}$ & .012 & $-.012^{\star}$ & .005 & .028 \\
\hline 34. Indirect (residence location $\rightarrow \mathrm{HC} \rightarrow \mathrm{BC}$ ) & 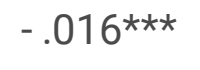 & .004 & $-.007 * \star \star$ & .002 & $<.001$ \\
\hline 35. Indirect (residence location $\rightarrow$ PF $\rightarrow$ BC) & .003 & .011 & .001 & .005 & .778 \\
\hline 36. Indirect (residence location $\rightarrow \mathrm{HC} \rightarrow \mathrm{PF} \rightarrow \mathrm{BC}$ ) & $-.014 * \star \star$ & .002 & $-.006 * \star \star$ & .001 & $<.001$ \\
\hline \multicolumn{6}{|c|}{$\begin{array}{l}\text { Note. S.E., standard error; } b \text {, unstandardized estimates; } \beta \text {, standardized estimates; } \mathrm{B} \text {, } \\
\text { bathing/showering; } \mathrm{D} \text {, dressing; } \mathrm{G} \text {, getting to the toilet; } M \text {, indoor mobility; } \mathrm{C} \text {, continence; } \mathrm{F} \text {, feeding; } \mathrm{HC} \text {, } \\
\text { health condition; } \mathrm{PF} \text {, physical functioning; FP, freedom from pain; } \mathrm{BC} \text {, being conscious. }{ }^{\star} p<.05,{ }^{\star \star} p< \\
.001,{ }^{\star \star \star} p<.001 \text {. }\end{array}$} \\
\hline
\end{tabular}

The results indicated that different dimensions of life prior to death predicted death qualities (i.e., painlessness and consciousness) to varying degrees. Freedom from diseases contributed to pain-free deaths to a moderately larger extent than to dying with consciousness $(|\beta| s=.194$ and .166 , both $p s<$ .001), but enhanced functioning capacity was associated more intensely with consciousness maintain rather than pain reduction $(|\beta| s=.387$ and .082 , both $p s<.001)$. Notably, the abovementioned, morbidity's effects on both quality markers of death were mediated by functional limitations: morbid individuals' tendency towards painful deaths was aggravated by problems they had with $\operatorname{ADLs}(|\beta|=.016, p<.001)$, 
and the amplitude of negative mediation of consciousness loss was even stronger at end-of-life $(|\beta|=$ $.077, p<.001)$. Nonetheless, both mediatory effects were smaller compared to the direct association between morbidity and a less-than-satisfactory death: the indirect effect of health condition on pain through physical functioning was minimal $(|\beta|=.016, p<.001)$ compared to the direct effect $(|\beta|=.177, p$ $<.001)$, although the respective indirect effect on consciousness was only slightly weaker than the direct one $(.077$ vs .089 , both $|\beta|$ s were statistically significant at $p=.001)$.

Older men and women took divergent trajectories of dying. Sex influenced physical functioning directly and indirectly through health condition: reported incidence of chronic diseases was slightly lower among older women than men $(|\beta|=.096, p<.001)$, and this modest health advantage only improved women's physical functioning trivially $(|\beta|=.019, p<.001)$. Given men's initial relatively pronounced advantage in functioning $(|\beta|=.120, p<.001)$, women still functioned worse than men $(|\beta|=.101, p<.001)$. These sex disparities indirectly impacted the quality of death: older women were less painful than men at death $(|\beta|$ $=.098, p<.001)$; yet men more often remained conscious $(|\beta|=.069, p<.001)$.

Compared to sex, residence location played a much lesser role during end-of-life. The rural dwellers appeared healthier $(|\beta|=.080, p<.001)$, which indirectly enhanced their functional capacities $(|\beta|=.016, p$ $<.001)$. However, location did not influence physical function directly $(|\beta|=.004, p=.778)$. On death quality, location had both direct and indirect effects regarding pain: rural residents were more prone to painful deaths than urban settlers $(|\beta|=.081, p<.001)$, but the former's better health marginally alleviated that effect to $|\beta|=.066(p<.001)$ because freedom from diseases marginally enhanced physical functioning, thereby relieving pain. Location did not directly affect consciousness when dying $(|\beta|=.021$, $p=.103)$, but given its impact on morbidity, as well as morbidity's effect on functional limitations $(|\beta|=$ $.012, p=.028)$, deaths in villages were slightly more associated with consciousness $(|\beta|=.033, p=.012)$.

\section{Discussion}

The current research was among the first known retrospective study investigating associations between life prior to death and quality of death based on sex and residence location among older Chinese. Three main lines of findings emerged.

First, almost two in every five older individuals in this research died less than satisfactorily, experiencing pain and/or loss of consciousness. Morbidity substantially increased chances of experiencing pain and unconsciousness when dying, and functional limitations caused by diseases moderately exacerbated such tendencies. Therefore, fundamental and foremost among the potential strategies for improving endof-life experiences and achieving 'a good death' is health management. After all, reducing morbidity has been proposed as a promising intervening target in successful aging [21,30]. Because most older adults

are community-dwelling in China, plausible measures to health management may include offering access to regular, community-based, and low-cost medical consultations and health examinations, as well as preventive home visits $[7,31]$. Additionally, provided that some older people already live with chronic diseases when facing proximity to death, reliable assistance in ADLs by professionals might be more 
beneficial to promoting their experiences during end-of-life. These findings on the dynamics of morbidity and functional difficulties, and their separate and joint impacts on death quality, should facilitate China's endeavour to improving its public healthcare reform, which is under strain due to a rapidly aging population. Of course, more research is required to address how community-based medical resources should be effectively utilized by those approaching death and their families, and how its budding end-oflife care services should be adequately developed.

Second, morbidity was moderately less prevalent among older women, which reduced their pain at death compared to men; older men's substantial advantage in physical functioning only mildly increased their likelihood of remaining conscious at death. This finding expounds the nuanced role sex plays given expected proximity to death, thus highlighting the need to consider sex-specific strategies when planning care and support for the aging population in China [13, 30,32]. Evidently, to improve end-of-life experiences, interventions that enhance functional capacity and lower incidence of chronic diseases should be respectively useful for women and men $[12,20]$.

Third, using rural-urban residence as a proxy for SES, analyses showed that compared to urban dwellers, rural residents were more prone to painful deaths, although this urban-rural divide was slightly narrowed by rural settlers' relative health. In fact, rural residents' preferable health state also indirectly led to their modest advantage in consciousness at death. These findings should alert policymakers and practitioners to the urban-rural disparities in access to medical resources and end-of-life care services: despite the lower likelihood of reported morbidity in rural areas, the settlers there were still at a higher risk of painful deaths than their peers in cities, suggesting unmet needs for pain management and symptom control among rural residents approaching deaths [30,33]. Furthermore, the government should address their divergent needs when they approach death: the former might require measures and resources to pain and symptom management, and the latter should capitalize on health-enhancing interventions to reduce morbidity $[6,19,30,34]$.

This research had two limitations. First, the end-of-life data in the CLHLS were confined to participants died between waves. Thereby, the level of representativeness of the sample for the target population (i.e., the deceased older adults across mainland China) on socio-demographic indicators was undetermined. Nonetheless, the mechanisms revealed linking life prior to death and quality of death should stand given the size of the sample. Second, the quality of death was ascertained by only two indicators reported by proxy respondents. Plausible disagreement between surrogate responses and those of the departed could have existed, and future research may adopt a prospective design to resolve this measurement issue. Still, this study enhances understandings of how the medical and functional dimensions of life prior to death affected different dimensions of death quality.

\section{Conclusions}

This retrospective study found that morbidity/multimorbidity and impaired physical functioning prior to death would curtail death quality. The finding that the former had a larger potential to increase pain when 
dying while the latter to reduce consciousness is essential, because it suggests that interventions addressing morbidity should be useful for older people at a higher risk of painful deaths (men and rural residents, for example) and those regarding functioning might be more needed by individuals prone to unconsciousness (such as women and urban dwellers).

\section{List Of Abbreviations}

\section{ADL}

activities of daily living

CFA

Confirmatory Factor Analysis

CFI

the comparative fit index

\section{CLHLS}

the Chinese Longitudinal Healthy Longevity Survey

CVD

cerebrovascular disease

\section{MIMIC method}

the 'multiple indicators, multiple causes' method

RMSEA

the root mean square error of approximation

\section{SEM}

Structural Equation Modelling

\section{SES}

socioeconomic status

\section{SRMR}

the standardized root mean residual

TLI

the Tucker-Lewis Index

\section{Declarations}

\section{Ethical approval and consent to participate}

The current study utilized data from the Chinese Longitudinal Healthy Longevity Survey (CLHLS), which was jointly approved by the Research Ethics Committee of Duke University (Pro00062871) and the Biomedical Ethics Committee of Peking University (IRB00001052-13074). The first author signed an online data use agreement (accessed from http://opendata.pku.edu.cn/) to obtain the CLHLS data. The authors confirm that all methods in this study were performed in accordance with relevant guidelines and regulations. 


\section{Consent for publication}

Not applicable because the dataset used in the study is publicly available.

\section{Availability of data and materials}

The dataset analyzed during the current study are available in the Peking University Open Access Research Database (https://doi.org/10.18170/DVN/WBO7LK).

\section{Competing interest}

The authors declare that they have no competing interests.

\section{Funding}

This work was supported by the Major Programme of National Fund of Philosophy and Social Science of China (20ZDZ077), and National Natural Science Foundation of China (71874031). The funding providers played no role in the design of the study, the analysis and interpretation of data, or writing of the current paper.

\section{Acknowledgements}

Not applicable.

\section{Author Contributions Statement}

$\mathrm{JL}$ analyzed and interpreted the data and wrote the first draft of the paper. LJS conceptualized the design and was a major contributor in writing the manuscript. XZP assisted in data analysis and the revising of the Results, Discussion, and Conclusion sections. ZH assisted in methodology, reviewing, and editing of the paper, and funding acquisition. All authors read and approved the final manuscript.

\section{References}

1. Patrick, DL. Measuring and Improving the Quality of Dying and Death. Ann Intern Med 2003;139:410. https://doi.org/10.7326/0003-4819-139-5_Part_2-200309021-00006

2. Pivodic L, De Burghgraeve T, Twisk J, van den Akker M, Buntinx F, Van den Block L. Changes in social, psychological, and physical well-being in the last 5 years of life of older people with cancer: A longitudinal study. Age and Ageing 2021;afab125. https://doi.org/10.1093/ageing/afab125

3. Lee HJ., Small BJ, Haley WE. Health and Well-Being in the Year before Death: The Association with Quality of Life and Care at the End-of-Life. J Aging Health 2020;32:1475-85. https://doi.org/10.1177/0898264320935297

4. Teno JM, Gozalo P, Trivedi AN, Bunker J, Lima J, Ogarek J, Mor V. Site of Death, Place of Care, and Health Care Transitions Among US Medicare Beneficiaries, 2000-2015. JAMA 2018;8:8981. 
https://doi.org/10.1001/jama.2018.8981

5. Steinhauser KE. Factors Considered Important at the End of Life by Patients, Family, Physicians, and Other Care Providers. JAMA 2000;284:2476. https://doi.org/10.1001/jama.284.19.2476

6. Gu D, Zeng Y. Healthiness of Survival and Quality of Death Among Oldest Old in China Using Fuzzy Sets. J Aging Health 2012;24:1091-130. https://doi.org/10.1177/0898264312453069

7. Dupre ME, Gu D, Warner DF, Yi Z. Frailty and type of death among older adults in China: prospective cohort study. BMJ 2009;338:b1175. https://doi.org/10.1136/bmj.b1175

8. Cheng G, Chen C. End-of-Life Needs of Dying Patients and Their Families in Mainland China: A Systematic Review. Omega (Westport). 2021 Feb 24;003022282199734. https://doi.org/10.1177/0030222821997340

9. Wiggins N, Droney J, Mohammed K, Riley J, Sleeman KE. Understanding the factors associated with patients with dementia achieving their preferred place of death: a retrospective cohort study. Age and Ageing 2019;48:433-9. https://doi.org/10.1093/ageing/afz015

10. Department of Economic and Social Affairs, United Nations. World population prospects: the 2012 revision. Volume II: demographic profiles. New York (US): UN DESA, Population Division; 2013.

11. Cui J, Mao F, Wang Z. Comorbidity of common chronic diseases among the elderly in China. Chinese Journal of Public Health. 2016 Jan;32:66-9. https://doi.org/10.11847/zgggws2016-32-01-20

12. Zhang W, Wei M. Estimating the level and duration of disability of the elderly in China: Based on the pooled data from multiple data sources. Population research 2015;39:3-14. https://doi.org/CNKI:SUN:RKYZ.0.2015-05-001

13. Alberts SC, Archie EA, Gesquiere LR, Altmann J, Vaupel JW, Christensen K. The Male-Female HealthSurvival Paradox: A Comparative Perspective on Sex Differences in Aging and Mortality. In: Weinstein M, Lane MA, editors. Sociality, Hierarchy, Health: Comparative Biodemography. Washington DC: National Academies Press; 2014. p. [339-63]. Available from: https://doi.org/10.17226/18822

14. Department of Aging and Life Course, World Health Organization. China country assessment report on aging and health. Geneva (CH): WHO Press; 2015.

15. Dai L, Ma J, Tao T. Impacts of chronic diseases on limited basic activities of daily living in middleaged and elderly people in China. Chin J Public Health 2018 Oct;34:1365-8.

16. Zhu H, Xie Y. Socioeconomic differentials in mortality among the oldest old in China. Research on Aging 2007;29:125-43. https://doi.org/10.1177/0164027506296758

17. Gu D, Yang F, Sautter J. Socioeconomic status as a moderator between frailty and mortality at old ages. BMC Geriatr 2016;16:151. https://doi.org/10.1186/s12877-016-0322-2

18. Zhao YW, Haregu TN, He L, Lu S, Katar A, Wang H, Yao Z, Zhang L. The effect of multimorbidity on functional limitations and depression amongst middle-aged and older population in China: $\mathrm{A}$ nationwide longitudinal study. Age and Ageing 2021;50:190-7. https://doi.org/10.1093/ageing/afaa117 
19. Zheng Z, Zhou Y. Quality of life before death of the elderly in China. Population and Economics 2019;233:44-54. https://doi.org/10.3969/j.issn.1000-4149.2019.02.004

20. Zhang Y, Lu J. Suffering or serene: An empirical study on determinants affecting the dying status of Chinese elderlies. Population and Development 2017;23:80-91.

21. Gu D, Liu G, Vlosky DA, Yi Z. Factors Associated with Place of Death Among the Chinese Oldest Old. Journal of Applied Gerontology 2007;36:34-57. https://doi.org/10.1177/0733464806296057

22. Gu D. General Data Quality Assessment of the CLHLS. In: Zeng Y, Poston DL, Vlosky DA, Gu D, editors. Healthy Longevity in China: Demographic Methods and Population Analysis, vol 20. Dordrecht; 2008. p. [39-60]. Available from: https://doi.org/10.1007/978-1-4020-6752-5

23. Zeng Y, Feng Q, Hesketh T, Christensen K, Vaupel JW. Survival, disabilities in activities of daily living, and physical and cognitive functioning among the oldest-old in China: a cohort study. The Lancet 2017;389:1619-29. https://doi.org/10.1016/S0140-6736(17)30548-2

24. Hair JF, Black WC, Babin BJ, Anderson RE. Multivariate data analysis. 7th edition. Harlow: Pearson new internat.; 2014. p. 583-4.

25. Woods CM. Evaluation of MIMIC-Model Methods for DIF Testing with Comparison to Two-Group Analysis. Multivariate Behavioural Research 2009;44:1-27. https://doi.org/10.1080/00273170802620121

26. Finch H. The MIMIC Model as a Method for Detecting DIF: Comparison with Mantel-Haenszel, SIBTEST, and the IRT Likelihood Ratio. Applied Psychological Measurement 2005;29:278-95. https://doi.org/10.1177/0146621605275728

27. Muthén, LK, Muthén, BO. Mplus User's Guide. 8th edition. Los Angeles: Muthén \& Muthén; 1998-2011.

28. George LK. Research design in end-of-life research: State of science. Gerontologist 2002;42:86-98. https://doi.org/10.1093/geront/42.suppl_3.86

29. Kline RB. Principles and Practice of Structural Equation Modelling. 4th edition. New York: the Guilford Press; 2016. p. 270-3.

30. Song L, Su C. Studies on the quality of death among the elderly in China: Current status and countermeasures. Social Sciences of Beijing 2021;6:119-28. https://doi.org/10.13262/j. bjsshkxy.bjshkx. 210611

31. Liimatta $\mathrm{H}$, Lampela P, Laitinen-Parkkonen P, Pitkala KH. Effects of preventive home visits on healthrelated quality-of-life and mortality in home-dwelling older adults. Scandinavian Journal of Primary Health Care 2019;37:90-97. https://doi.org/10.1080/02813432.2019.1569372

32. Oksuzyan A, Petersen I, Stovring H, Bingley P, Vaupel JW, Christensen K. The Male-Female HealthSurvival Paradox: A Survey and Register Study of the Impact of Sex-Specific Selection and Information Bias. Annals of Epidemiology 2009;19:504-11.

https://doi.org/10.1016/j.annepidem.2009.03.014

33. Ma S, Zhou X, Jiang M, Li Q, Gao C, Cao W, Li L. Comparison of access to health services among urban-to-urban and rural-to-urban older migrants, and urban and rural older permanent residents in 
Zhejiang Province, China: A cross-sectional survey. BMC Geriatr 2018;18:174.

https://doi.org/10.1186/s12877-018-0866-4

34. Liang J, McCarthy JF, Jain A, Krause N, Bennett JM, Gu S. Socioeconomic Gradient in Old Age Mortality in Wuhan, China. The Journals of Gerontology Series B: Psychological Sciences and Social Sciences 2000;55:S222-S233. https://doi.org/10.1093/geronb/55.4.S222

\section{Figures}

Panel A. CFA model of physical functioning

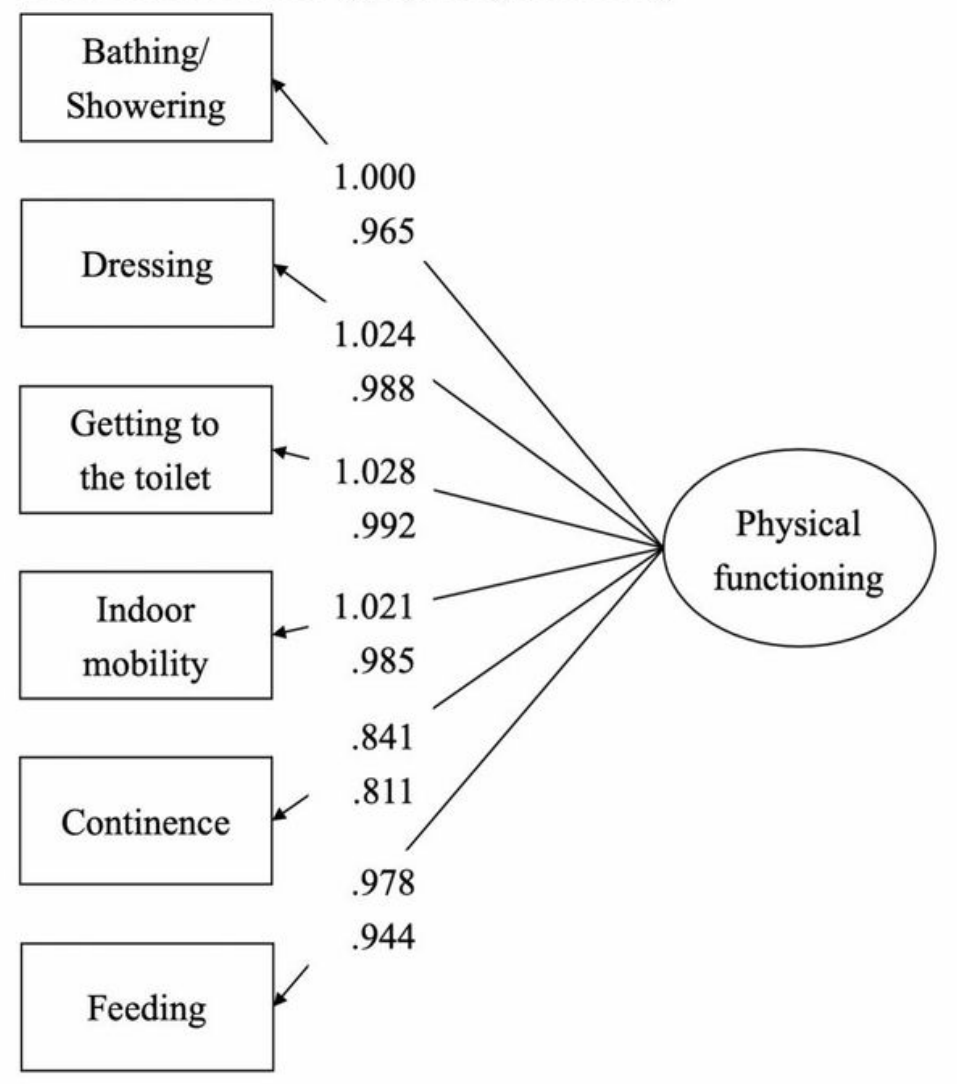

Panel B. MIMIC model linking gender to bathing/showering

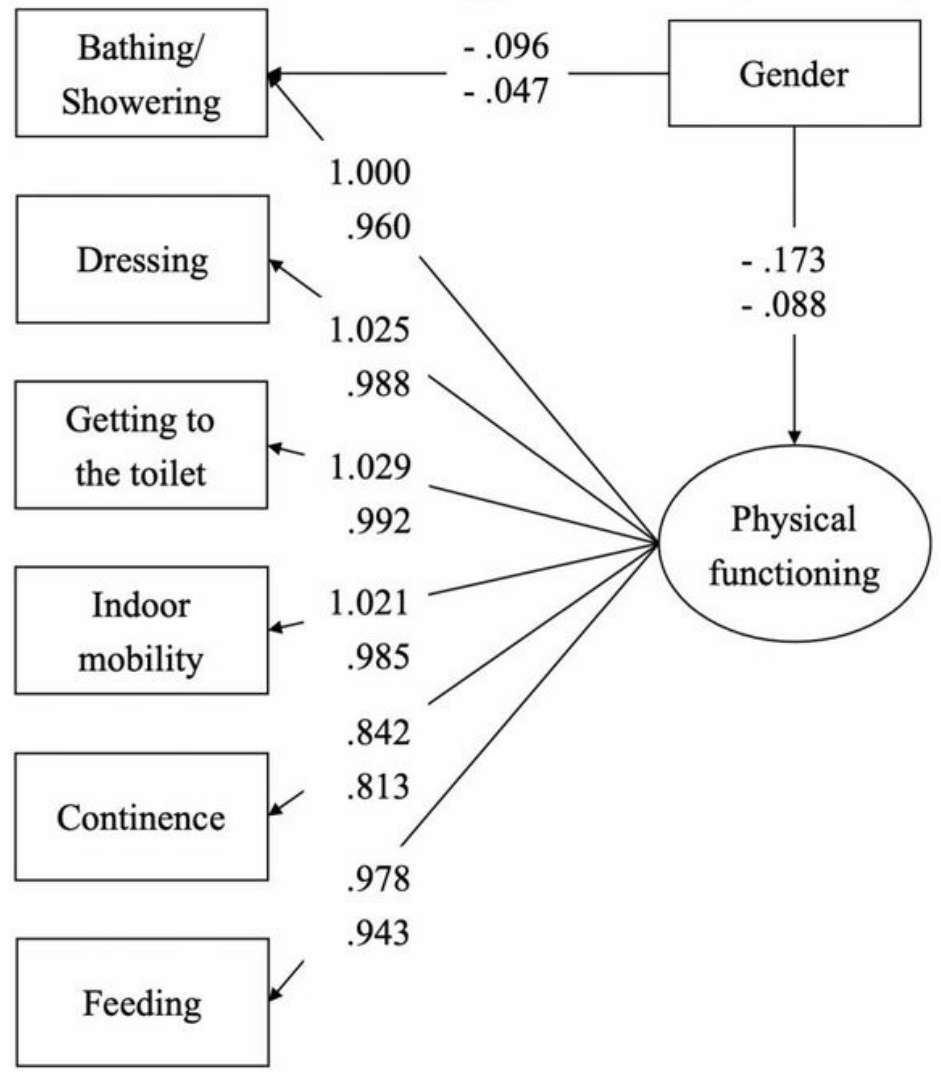

\section{Figure 1}

CFA model of physical functioning and example MIMIC model linking sex to bath/showering

Note. Both unstandardized (upper) and standardized estimates (lower) are presented. Fit indices for CFA model (Panel A): $c^{2}(\mathrm{df})=135.319$ (9), $p<.001 ; \mathrm{CFI}=1.000 ; \mathrm{TLI}=1.000 ; \mathrm{RMSEA}=.038,90 \%$ Cl: [.033, .044]; SRMR $=.010$. Fit indices for MIMIC model (Panel B): $c^{2}(\mathrm{df})=170.292(13), p<.001 ; \mathrm{CFI}=1.000 ; \mathrm{TLI}=$ $.999 ; \mathrm{RMSEA}=.036,90 \% \mathrm{Cl}$ [.031, .040]; SRMR $=.013$. 


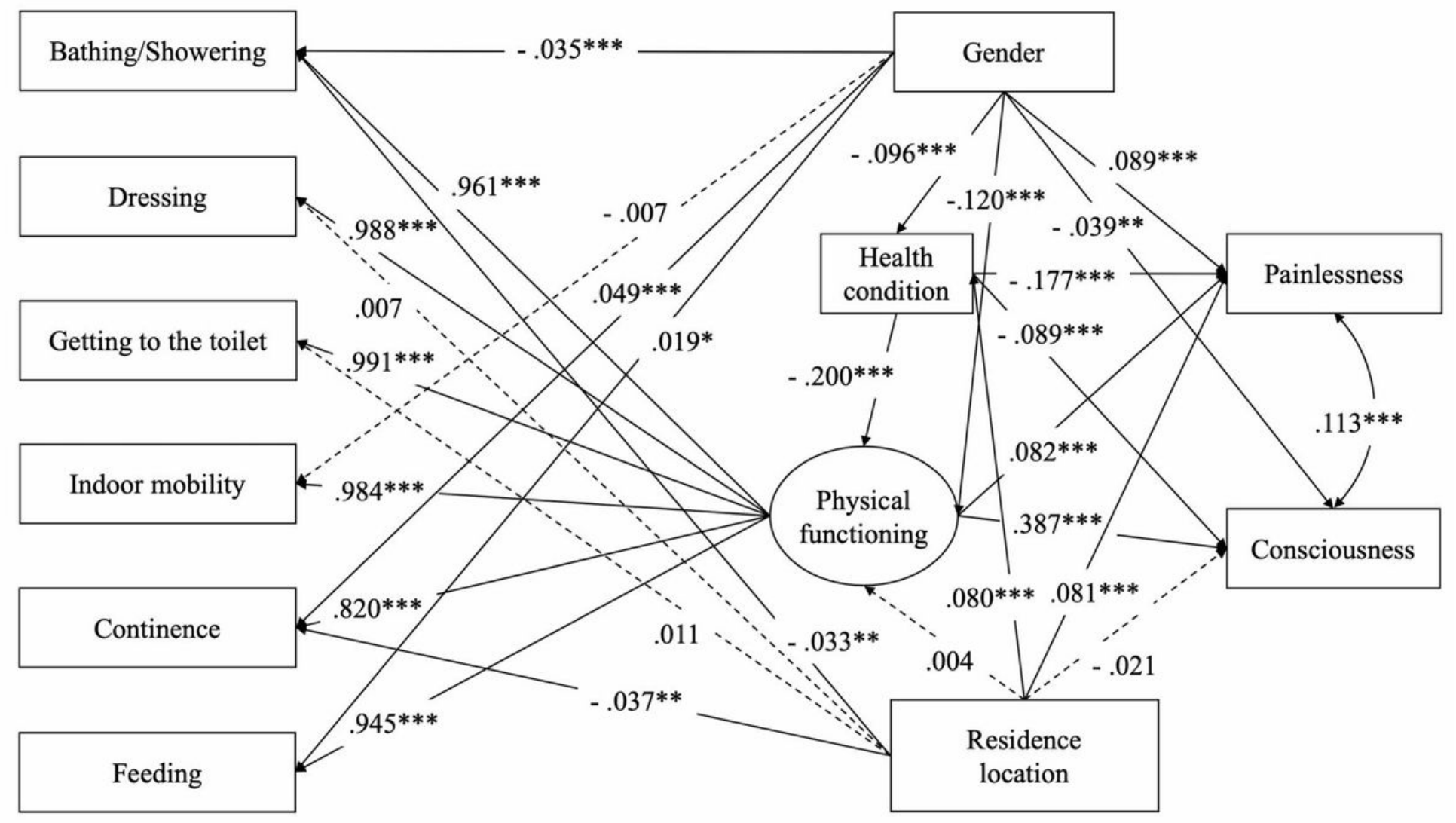

Figure 2

SEM model of associations between life prior to death and quality of death

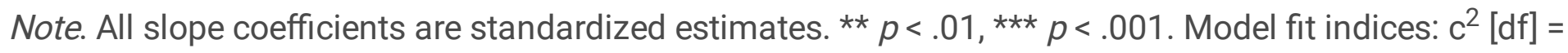
293.502 [26], $p<.001 ; \mathrm{CFI}=.999 ; \mathrm{TLI}=.999 ; \mathrm{RMSEA}=.033,90 \% \mathrm{Cl}:[.029, .036]$; SRMR $=.015$. 